\title{
Differences in the genetic mechanism of the response to stress between wheat varieties
}

\author{
Konstantinov D.K. ${ }^{1,2 *}$, Ermakov A.A. ${ }^{1}$, Bobrovskikh A.V. ${ }^{1,2}$, Zubairova U.S. ${ }^{1,2}$, \\ Doroshkov A.V. ${ }^{1,2}$ \\ ${ }^{1}$ Institute of Cytology and Genetics, SB RAS, Novosibirsk, Russia \\ ${ }^{2}$ Novosibirsk State University, Novosibirsk, Russia \\ *e-mail: konstantinov@bionet.nsc.ru
}

Motivation: Triticum aestivum is one of the most important agricultural plants. Abiotic stresses such as drought and cold are a common cause of reduced productivity of this culture. Resistance to abiotic factors is a complex (multigenic) traits. The study of the stress response mechanisms of $T$. aestivum is an actual goal of basic research and has practical significance. To identify a complete set of genes an analysis of full transcriptome data is necessary. Transcriptome sequencing was performed using MACE method under control and stress conditions.

Method and algorithm: In this work we used plants of bread wheat (Triticum aestivum L.) varieties Saratovskaya 29 and Yanetskis Probat with different abiotic stress tolerance. The plants were planted in mobile hydroponic pots and grown under controlled day/night cycle (16/8 hour). Cold stress treatment were simulated in a climatic chamber at $+4{ }^{\circ} \mathrm{C}$ for 6 and 24 hours with the same lighting conditions. Drought simulated by discontinuation of watering. After treatments tissue samples were sampled, freezed in liquid nitrogen and stored at $-80{ }^{\circ} \mathrm{C}$. Sequencing was performed on Illumina NexSeq 500 platform. The FastQC program was used to analyze the quality of the libraries. Trimmomatic was used to filter the libraries. STAR was used to mapping reads to reference genome. Search for the differentially expressed genes was performed by featureCounts from package subread-1.6.3-source and EdgeR.

Results: Analysis of T. aestivum transcriptomic data revealed 1292 genes differentially expressed under stress conditions. Most of the genes are differentially expressed in cold. The gene lists of cold-response between the studied varieties are intersect more strongly. Differences in the mechanisms of reaction to stress between the studied varieties were revealed. Identified genes can be used in subsequent genome selection.

Acknowledgements: Supported by grant from RSF No. 17-74-10198. 